\title{
Ischemic Stroke in a 29-Year-Old Patient with COVID-19: A Case Report
}

\author{
Christian Avvantaggiato a, b Loredana Amoruso $^{a} \quad$ Maria Pia Lo Muzio $^{a}$ \\ Maria Assunta Mimmo ${ }^{a}$ Michelina Delli Bergoli ${ }^{a} \quad$ Nicoletta Cinone ${ }^{a}$ \\ Luigi Santoro $^{a}$ Lucia Stuppiello $^{a}$ Antonio Turitto ${ }^{a}$ Chiara Ciritella ${ }^{a}$ \\ Pietro Fiore $^{b, c}$ Andrea Santamato ${ }^{a}$ \\ aSpasticity and Movement Disorders "ReSTaRt" Unit, Physical Medicine and Rehabilitation \\ Section, OORR Hospital, University of Foggia, Foggia, Italy; 'bstituti Clinici Scientifici \\ Maugeri, IRCCS Institute of Bari, Bari, Italy; ' Department of Clinical and Experimental \\ Medicine, University of Foggia, Foggia, Italy
}

Keywords

Stroke · Coronavirus disease 2019 - SARS-CoV-2 · Cerebral infarction · Hypercoagulability

\begin{abstract}
Increasing evidence reports a greater incidence of stroke among patients with Coronavirus disease 2019 (COVID-19) than the non-COVID-19 population and suggests that SARS-CoV-2 infection represents a risk factor for thromboembolic and acute ischemic stroke. Elderly people have higher risk factors associated with acute ischemic stroke or embolization vascular events, and advanced age is strongly associated with severe COVID-19 and death. We reported, instead, a case of an ischemic stroke in a young woman during her hospitalization for COVID-19-related pneumonia. A 29-year-old woman presented to the emergency department of our institution with progressive respiratory distress associated with a 2-day history of fever, nausea, and vomiting. The patient was transferred to the intensive care unit (ICU) where she underwent a tracheostomy for mechanical ventilation due to her severe clinical condition and her very low arterial partial pressure of oxygen. The nasopharyngeal swab test confirmed SARS-CoV-2 infection. Laboratory tests showed neutrophilic leucocytosis, a prolonged prothrombin time, and elevated D-dimer and fibrinogen levels. After 18 days, during her stay in the ICU after suspension of the medications used for sedation, left hemiplegia was reported. Central facial palsy on the left side, dysarthria, and facial drop were present, with complete paralysis of the ipsilateral upper and lower limbs. Computed tomography (CT) of the head and magnetic resonance imaging of the brain confirmed the presence of lesions in the right hemisphere affecting the territories of the anterior and middle cerebral arteries, consistent with ischemic stroke. Pulmonary and splenic infarcts were also found after CT of the chest. The age of the patient and the absence of serious concomitant cardiovascular diseases place the emphasis on the capacity of SARS-CoV-2 infection to be an independent cerebro-
\end{abstract}


vascular risk factor. Increased levels of D-dimer and positivity to $\beta 2$-glycoprotein antibodies could confirm the theory of endothelial activation and hypercoagulability, but other mechanisms - still under discussion - should not be excluded.

(C) 2021 The Author(s).

Published by S. Karger AG, Basel

\section{Background}

Coronavirus disease 2019 (COVID-19), caused by the novel coronavirus SARS-CoV-2, is characterized by a wide range of symptoms, most of which cause acute respiratory distress syndrome [1, 2], associated with intensive care unit (ICU) admission and high mortality [3]. On March 11, 2020, the large global outbreak of the disease led the World Health Organization (WHO) to declare COVID-19 a pandemic, with 11,874,226 confirmed cases and 545,481 deaths worldwide (July 9, 2020) [4]. In many cases, the clinical manifestations of COVID-19 are characteristic of a mild disease that may, however, worsen to a critical lower respiratory infection [2]. At the onset of the disease, the most frequent symptoms are fever, dry cough, fatigue, and shortness of breath as the infection progresses may appear signs and symptoms of respiratory failure that require ICU admission [5, 6]. Although acute respiratory distress syndrome is the most important cause of ICU admission for COVID-19 patients, several studies have underlined the presence of neurological symptoms such as confusion, dizziness, impaired consciousness, ataxia, seizure, anosmia, ageusia, vision impairment, and stroke $[7,8]$. In particular, the state of hypercoagulability in patients affected by COVID-19 favors the formation of small and/or large blood clots in multiple organs, including the brain, potentially leading to cerebrovascular disease (ischemic stroke but also intracranial hemorrhage) [9, $10]$.

We found an interesting case of stroke following a SARS-CoV-2 infection in a young patient. A 29-year-old woman, during her ICU hospitalization for COVID-19-related pneumonia, was diagnosed with ischemic stroke of the right hemisphere, without other cardiac/ cerebrovascular risk factors except hypertension. The young age of the patient and the absence of higher cerebrovascular risk factors make the present case very interesting as it can help demonstrate that COVID-19 is an independent risk factor for acute ischemic stroke. In a case series of 214 patients with COVID-19 (mean [SD] age, 52.7 [15.5] years), neurologic symptoms were more common in patients with severe infection who were older than the others [11]. New-onset CVD was more common in COVID-19 patients who had underlying cerebrovascular risk factors, such as older age ( $>65$ years) [12], and very few cases of stroke in patients younger than 50 years have been reported $[12,13]$. Our case seems to be the only one younger than 30 years.

\section{Case Presentation}

On the night between March 19 and 20, 2020, a 29-year-old woman was referred to our hospital "Policlinico Riuniti di Foggia" due to a progressive respiratory distress associated with a 2-day history of fever, nausea, and vomiting. At presentation, the heart rate was 128 $\mathrm{bpm}$, the blood oxygen saturation measured by means of the pulse oximeter was $27 \%$, the respiratory rate was 27 breaths per minute, and the blood pressure was 116/77 $\mathrm{mm} \mathrm{Hg}$. The arterial blood gas test showed a $\mathrm{pH}$ of 7.52, $\mathrm{pO}_{2} 20 \mathrm{~mm} \mathrm{Hg}$, and $\mathrm{pCO}_{2} 34 \mathrm{~mm} \mathrm{Hg}$. The patient was immediately transferred to the ICU where she underwent tracheostomy and endotracheal intubation for mechanical ventilation due to her severe clinical condition and deterio-

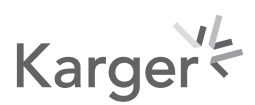


rated pulmonary gas exchange. The diagnosis of COVID-19 was confirmed by PCR on a nasopharyngeal swab.

The family medical history was normal, and the only known pre-existing medical conditions were polycystic ovary syndrome (diagnosed 3 years earlier), conversion disorder, and hypertension (both diagnosed 2 years earlier). Ramipril and nebivolol were prescribed for the high blood pressure treatment, and sertraline was prescribed for the conversion disorder treatment. Drug therapy adherence was inconstant. The patient had no history of diabetes, cardiac pathologies, strokes, transient ischemic attacks, thromboembolic, or other vascular pathologies.

Laboratory tests showed neutrophilic leukocytosis (white blood cell count $14.79 \times 10^{3}$, neutrophil percentage $89.8 \%$, and neutrophil count $13.29 \times 10^{3}$ ), a prolonged prothrombin time (15.3 s) with a slightly elevated international normalized ratio (1.38), and elevated D-dimer $(6,912 \mathrm{ng} / \mathrm{mL})$ and fibrinogen levels $(766 \mathrm{mg} / \mathrm{dL})$. Other findings are shown in Table 1.

Table 1. Laboratory test

\begin{tabular}{|c|c|c|}
\hline & 20 March 2020 & 7 April 2020 \\
\hline RBC count, $\times 10^{6}$ & $\downarrow 3.66$ & $\downarrow 3.18$ \\
\hline Hemoglobin, g/dL & $\downarrow 10.7$ & $\downarrow 9.3$ \\
\hline Hematocrit, \% & $\downarrow 30.9$ & $\downarrow 28.0$ \\
\hline White blood cell count, $\times 10^{3}$ & $\uparrow 14.79$ & 9.17 \\
\hline Neutrophils, \% & $\uparrow 89.8$ & $\uparrow 81.2$ \\
\hline Lymphocytes, \% & 6.7 & 12.9 \\
\hline Monocytes, \% & 3.2 & 5.1 \\
\hline Eosinophils, \% & 0.1 & 0.5 \\
\hline Basophiles, \% & 0.2 & 0.3 \\
\hline Prothrombin time & $15.3 \mathrm{~s} ; 62 \%$ & $14.7 \mathrm{~s} ; 66 \%$ \\
\hline INR & $\uparrow 1.38$ & $\uparrow 1.33$ \\
\hline Partial thromboplastin time & 27.7 & 32.7 \\
\hline Fibrinogen, mg/dL & $\uparrow 766$ & $\uparrow 463$ \\
\hline Antithrombin, $\%$ & 89 & 108 \\
\hline D-dimer, ng/mL & $\uparrow 6,912$ & $\uparrow 4,961$ \\
\hline Epathic function & $\mathrm{OK}$ & Gamma gt $91 \mathrm{U} \mathrm{L}$ \\
\hline Glucose, mg/dL & $\uparrow 216$ & $\uparrow 160$ \\
\hline Pancreatic function & $\mathrm{OK}$ & $\mathrm{OK}$ \\
\hline HS troponin 1, ng/L & 128.1 & 128.1 \\
\hline Adrenaline plasma, ng/L & ND & 9 \\
\hline ENA6, U/mL & ND & 2.0 \\
\hline ANCA, $\mathrm{U} / \mathrm{mL}$ & ND & 2.0 \\
\hline Anticardiolipin IgG, U/mL & ND & 12.9 \\
\hline Anticardiolipin IgM, U/mL & ND & 11.7 \\
\hline Anti-beta2 glycoprotein IgG, U/mL & ND & 5.0 \\
\hline Anti-beta2 glycoprotein IgM, U/mL & ND & +15.4 \\
\hline
\end{tabular}

INR, international normalized ratio. 
This pharmacological therapy was set as follows: enoxaparin 6,000 U.I. once a day, piperacillin $4 \mathrm{~g} /$ tazobactam $0.5 \mathrm{~g}$ twice a day; Kaletra, a combination of lopinavir and ritonavir indicated for human immunodeficiency virus (HIV) infection treatment, 2 tablets twice a day; hydroxychloroquine $200 \mathrm{mg}$ once a day; and furosemide $250 \mathrm{mg}$, calcium gluconate, and aminophylline $240 \mathrm{mg} 3$ times a day. No adverse events were reported.

On April 7, 2020, during her stay in the ICU and after suspension of the medications used for sedation, left hemiplegia was reported. The same day, the patient underwent a computed tomography examination of the head, which showed areas of hypodensity in the right hemisphere due to recent cerebral ischemia.

On April 16, 2020, the patient was oriented to time, place, and person. Central facial palsy on the left side, dysarthria, and facial drop were present, with complete paralysis of the ipsilateral upper and lower limbs. The power of all the muscles of the left limbs was grade 0 according to the Medical Research Council (MRC) scale. Deep tendon reflexes were reduced on the left upper limb but hyperactive on the ipsilateral lower limb, with a slight increase in the muscle tonus. The senses of touch, vibration, and pain were reduced on the left side of the face and body.

On the same day, the patient underwent magnetic resonance imaging (MRI) of the brain (Fig. 1a), showing lesions on the right hemisphere affecting the territories of the anterior and middle cerebral arteries. On May 5, 2020, magnetic resonance angiography showed an early duplication of the sphenoidal segment of the right middle cerebral artery, the branches of which are irregular with rosary bead-like aspects (Fig. 1d, e); on the same day, the second MRI
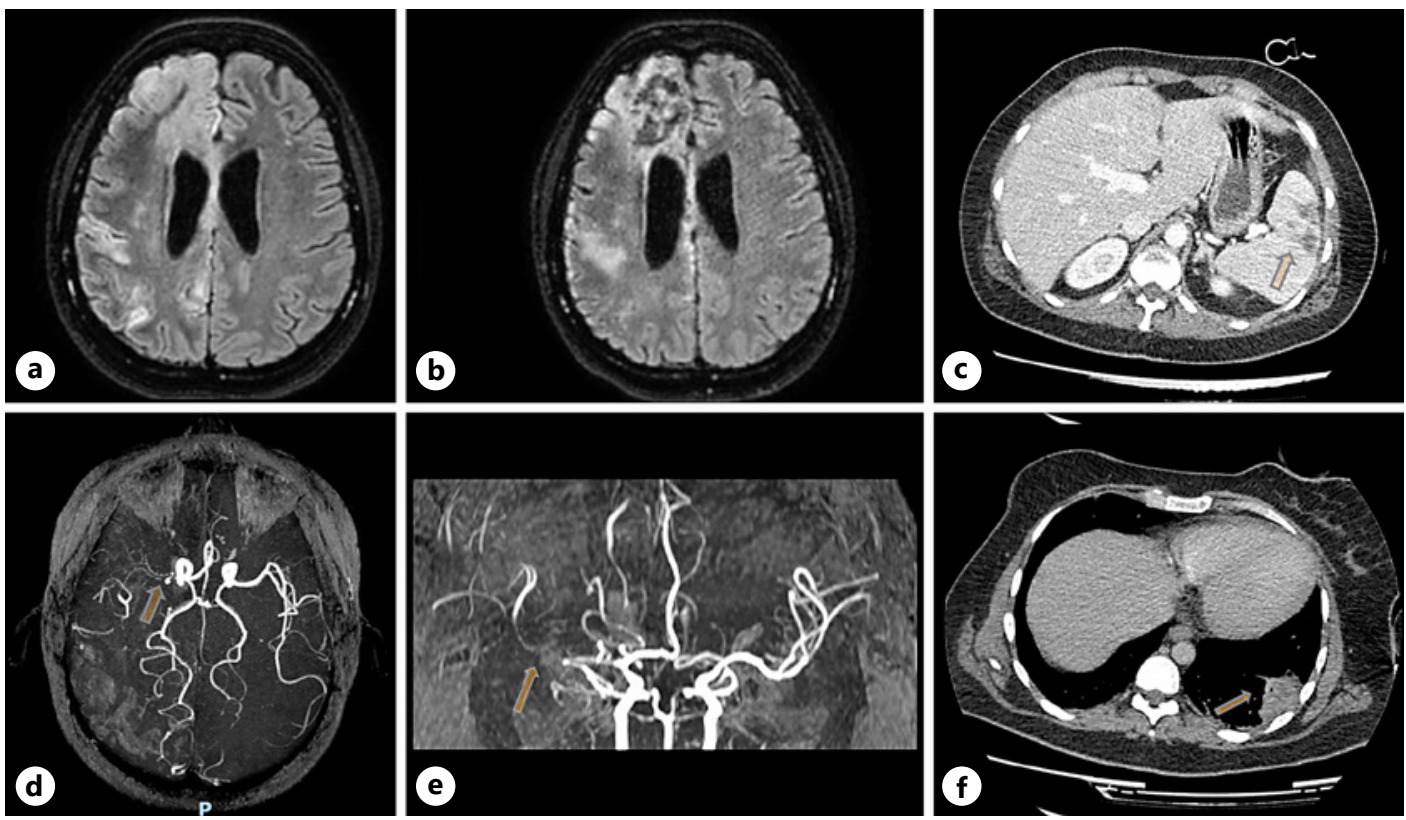

Fig. 1. Imaging. a April 16, 2020; MRI of the brain: lesions in the right hemisphere affecting the territories of the anterior and the middle cerebral arteries. b May 5, 2020; MRI of the brain: same lesions in the right hemisphere shown in the previous image. d, e May 5, 2020; MRA showed an early duplication of the sphenoidal segment of the right middle cerebral artery, the branches of which are irregular with rosary beadlike aspect and reduction of blood flow in the middle cerebral artery. c April 20, 2020; CT of the abdomen: vascular lesion, consistent with a splenic infarct, found in the inferior pole of the spleen. $\mathbf{f}$ April 20, 2020; CT of the chest: basal subpleural area of increased density within the left lung $(4 \times 4 \times 3 \mathrm{~cm})$, consistent with a pulmonary infarction. MRA, magnetic resonance angiography; CT, computed tomography; MRI, magnetic resonance imaging. 
(Fig. 1b) confirmed the lesions. Computed tomography of the chest (Fig. 1c) and abdomen (Fig. 1f), performed 5 days after the MRI of the brain, showed not only multifocal bilateral ground-glass opacities but also a basal subpleural area of increased density within the left lung $(4 \times 4 \times 3 \mathrm{~cm})$, consistent with a pulmonary infarction. In addition, a vascular lesion, consistent with a splenic infarct, was found in the inferior pole of the spleen. Doppler echocardiography of the hearth showed regular right chambers and left atrium and a slightly hypertrophic left ventricle with normal size and kinetics (ejection fraction: 55\%). The age of the patientand the absence of serious concomitant cardiovascular diseases place the emphasis on the capacity of SARS-CoV-2 infection to be an independent cerebrovascular risk factor.

\section{Discussion}

The pandemic outbreak of novel SARS-CoV-2 infection has caused great concern among the services and authorities responsible for public health due to not only the mortality rate but also the danger of filling up hospital capacities in terms of ICU beds and acute non-ICU beds. In this regard, the nonrespiratory complications of COVID-19 should also be taken into great consideration, especially those that threaten patients' lives and extend hospitalization times. Stroke is one of these complications, since a greater incidence of stroke among patients with COVID-19 than the non-COVID-19 population has been reported, and a preliminary casecontrol study demonstrated that SARS-CoV-2 infection represents a risk factor for acute ischemic stroke [14].

We found that the reported case is extremely interesting, since the woman is only 29 years old and considering how stroke in a young patient without other known risk factors is uncommon. Not only elderly people have higher risk factors associated with acute ischemic stroke or embolization vascular events [15], but it is also true that advanced age is strongly associated with severe COVID-19 and death. The severity of the disease is directly linked to immune dysregulation, cytokine storm, and acute inflammation state, which in turn are more common in patients who present immunosenescence [6].

Inflammation plays an important role in the occurrence of cardiovascular and cerebrovascular diseases since it favors atherosclerosis and affects plaque stability [16]. The ischemic stroke of the 29-year-old woman does not appear to be imputable to emboli originating a preexisting atheromatous plaque, both for the age of the patient and for the absence of plaques at the Doppler ultrasound study of the supra-aortic trunks.

Most likely, COVID-19-associated hypercoagulability and endothelial dysfunction are the causes of ischemic stroke, as suggested by other studies and case reports $[10,13,17]$. Although the mechanisms by which SARS-CoV-2 infection leads to hypercoagulability are still being studied, current knowledge suggests that cross talk between inflammation and thrombosis has a crucial role [18]. The release of inflammatory cytokines leads to the activation of epithelial cells, monocytes, and macrophages. Direct infection of endothelial cells through the ACE2 receptor also leads to endothelial activation and dysfunction, expression of tissue factor, and platelet activation and increased levels of VWF and FVIII, all of which contribute to thrombin generation and fibrin clot formation [17]. The 29-year-old patient showed an increased level of D-dimer, which is a degradation product of cross-linked fibrin, indicating a global activation of hemostasis and fibrinolysis and conforming to the hypothesis of COVID-19-associated hypercoagulability. Endothelial activation and hypercoagulability are also confirmed by positivity to $\beta 2$ glycoprotein antibodies. Anticardiolipin antibody and/or $\beta 2$ glycoprotein antibody positivity has been reported in a few studies $[17,19,20]$. In addition, widespread thrombosis in SARS-CoV-2 infection could also be caused by neutrophil extracellular traps (NETs). Neutrophilia [21] and an elevated neutrophil-lymphocyte ratio [22] have been reported by numerous

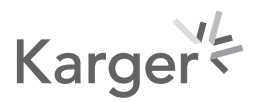


studies as predictive of worse disease outcomes, and recently, the contribution of NETs in the pathophysiology of COVID-19 was reported [23]. Thrombogenic involvement of NETs has been described in various settings of thrombosis, including stroke, myocardial infarction, and deep vein thrombosis [24]. The high neutrophil count found in our case does not exclude the hypothesis that NETs are involved in the pathogenesis of ischemic stroke.

\section{Conclusion}

Ischemic stroke in young patients without pre-existing cerebrovascular risk factors is very unusual. In this regard, our case of an ischemic stroke, reported in a 29-year-old woman, is very interesting. Although it is not possible to determine precisely when the thromboembolic event occurred, our case of stroke during COVID-19-related pneumonia seems to confirm that COVID-19 is an independent risk factor for acute ischemic stroke. The mechanisms by which coronavirus disease leads to stroke are still under study, but it is clear that hypercoagulability and endothelial activation play a key role. Testing for SARS-CoV-2 infection should be considered for patients who develop neurologic symptoms, but it is equally important to monitor COVID-19 patients during their hospitalization to find any neurological sign or symptom in a timely manner. Our case suggests that discovering neurological deficits in sedated patients promptly can be very difficult; for this reason, sedation in mechanically ventilated patients has to be considered only if strictly necessary. Performing serial laboratory testing and waking up the patient as soon as clinical conditions allow are strategies that should be taken into account.

\section{Statement of Ethics}

Written informed consent was obtained from the patient for publication of this case report and any accompanying images. A copy of the written consent is available for review by the editor-in-chief of this journal.

\section{Conflict of Interest Statement}

The authors certify that there is no conflict of interest with any financial organization regarding the material discussed in the manuscript.

\section{Funding Sources}

No funding was received for the publication of this case report.

\section{Author Contributions}

All authors agree with the contents of the manuscript and were fully involved in the study and preparation of the manuscript. All authors read and approved the final version of the manuscript. M.A. Mimmo, M.P. Lo Muzio, M. Delli Bergoli, and L. Amoruso collected the data. C. Avvantaggiato wrote the manuscript with support of N. Cinone, L. Santoro, and C. Ciritella. C. Avvantaggiato, A. Turitto, and L. Stuppiello researched and discussed the neurophysiological principles of this study. P. Fiore and A. Santamato supervised the project. 


\section{References}

1 Lai CC, Shih TP, Ko WC, Tang HJ, Hsueh PR. Severe acute respiratory syndrome coronavirus 2 (SARS-CoV-2) and coronavirus disease-2019 (COVID-19): the epidemic and the challenges. Int J Antimicrob Agents. 2020 [cited 2020 Jul 9];55(3):105924. Available from: /pmc/articles/PMC7127800/?report=abstract

2 Ortiz-Prado E, Simbaña-Rivera K, Gómez-Barreno L, Rubio-Neira M, Guaman LP, Kyriakidis NC, et al. Clinical, molecular and epidemiological characterization of the SARS-CoV2 virus and the coronavirus disease 2019 (COVID-19), a comprehensive literature review. Diagn Microbiol Infect Dis. 2020 May [cited 2020 Jul 9];98(1): 115094. Available from: /pmc/articles/PMC7260568/?report=abstract

3 Huang C, Wang Y, Li X, Ren L, Zhao J, Hu Y, et al. Clinical features of patients infected with 2019 novel coronavirus in Wuhan, China. Lancet. 2020 Feb 15 [cited 2020 Jul 9];395(10223):497-506. Available from: https:// isaric.tghn.org/protocols/

4 WHO Coronavirus Disease (COVID-19) Dashboard|WHO Coronavirus Disease (COVID-19) Dashboard [Internet] [cited 2020 Jul 10]. Available from: https://covid19.who.int/

5 Symptoms of Coronavirus|CDC [Internet] [cited 2020 Jul 10]. Available from: https://www.cdc.gov/ coronavirus/2019-ncov/symptoms-testing/symptoms.html

6 Huang C, Wang Y, Li X, Ren L, Zhao J, Hu Y, et al. Clinical features of patients infected with 2019 novel coronavirus in Wuhan, China. Lancet. 2020 Feb 15 [cited 2020 Jul 10];395(10223):497-506. Available from: https: //isaric.tghn.org/protocols/

7 Mao L, Jin H, Wang M, Hu Y, Chen S, He Q, et al. Neurologic manifestations of hospitalized patients with coronavirus disease 2019 in Wuhan, China. JAMA Neurol. 2020 Jun 1 [cited 2020 Jul 10];77(6):683-90. Available from: https://jamanetwork.com/

8 Li Z, Liu T, Yang N, Han D, Mi X, Li Y, et al. Neurological manifestations of patients with COVID-19: potential routes ofSARS-CoV-2 neuroinvasion from the periphery to the brain. Front Med.2020;14(5):533-41. Available from: https://doi.org/10.1007/s11684-020-0786-5

9 Spiezia L, Boscolo A, Poletto F, Cerruti L, Tiberio I, Campello E, et al. COVID-19-related severe hypercoagulability in patients admitted to intensive care unit for acute respiratory failure. Thromb Haemost. 2020 Jun 1 [cited 2020 Jul 10];120(6):998-1000. Available from:/pmc/articles/PMC7295272/?report=abstract

10 Fotuhi M, Mian A, Meysami S, Raji CA. Neurobiology of COVID-19. J Alzheimers Dis. 2020 Jun 9;76(1):3-19.

11 Mao L, Jin H, Wang M, Hu Y, Chen S, He Q, et al. Neurologic manifestations of hospitalized patients with coronavirus disease 2019 in Wuhan, China. JAMA Neurol. 2020 [cited 2020 Jul 29];77(6):1. Available from: /pmc/ articles/PMC7149362/?report=abstract

12 Reddy ST, Reddy ST, Garg T, Shah C, Nascimento FA, Imran R, et al. Cerebrovascular disease in patients with COVID-19: a review of the literature and case series. Case Rep Neurol. 2020 [cited 2020 Jul 29];12(2):199209. Available from: /pmc/articles/PMC7325208/?report=abstract

13 Oxley TJ, Mocco J, Majidi S, Kellner CP, Shoirah H, Singh IP, et al. Large-vessel stroke as a presenting feature of Covid-19 in the young. N Engl J Med. 2020 May 14 [cited 2020 Jul 18];382(20):e60. Available from: http:// www.nejm.org/doi/10.1056/NEJMc2009787

14 Belani P, Schefflein J, Kihira S, Rigney B, Delman BN, Mahmoudi K, et al. COVID-19 is an independent risk factor for acute ischemic stroke. AJNR Am J Neuroradiol. 2020;41:1361-4.

15 The impact of COVID-19 on ischemic stroke: a case report. 2020 Mar 31 [cited 2020 Jul 18]. Available from: https://www.researchsquare.com/article/rs-20393/v1

16 Hartmann P, Schober A, Weber C. Chemokines and microRNAs in atherosclerosis. Cell Mol Life Sci. 2015 [cited 2020 Jul 18];72(17):3253-66. Available from: https://pubmed.ncbi.nlm.nih.gov/26001902/

17 Abou-Ismail MY, Diamond A, Kapoor S, Arafah Y, Nayak L. The hypercoagulable state in COVID-19: incidence, pathophysiology, and management. Thromb Res. 2020 [cited 2020 Jul 16];194:101-15. Available from: /pmc/ articles/PMC7305763/?report=abstract

18 Fotuhi M, Mian A, Meysami S, Raji CA. Neurobiology of COVID-19. J Alzheimers Dis. 2020;76:3-19.

19 Helms J, Tacquard C, Severac F, Leonard-Lorant I, Ohana M, Delabranche X, et al. High risk of thrombosis in patients with severe SARS-CoV-2 infection: a multicenter prospective cohort study. Intensive Care Med. 2020 Jun 1 [cited 2020 Jul 16];46(6):1089-98. Available from: https://doi.org/10.1007/s00134-020-06062-x

20 Escher R, Breakey N, Lämmle B. Severe COVID-19 infection associated with endothelial activation. Thromb Res. 2020;190:62.

21 Wang D, Hu B, Hu C, Zhu F, Liu X, Zhang J, et al. Clinical characteristics of 138 hospitalized patients with 2019 novel coronavirus-infected pneumonia in Wuhan, China. JAMA. 2020 Mar 17 [cited 2020 Jul 16];323(11): 1061-9. Available from: https://jamanetwork.com/

22 Liu J, Liu Y, Xiang P, Pu L, Xiong H, Li C, et al. Neutrophil-to-lymphocyte ratio predicts severe illness patients with 2019 novel coronavirus in the early stage. medRxiv. 2020 Feb 12 [cited 2020 Jul 16]. Available from: https://doi.org/10.1101/2020.02.10.20021584

23 Zuo Y, Yalavarthi S, Shi H, Gockman K, Zuo M, Madison JA, et al. Neutrophil extracellular traps in COVID-19. JCI Insight. 2020 Jun 4 [cited 2020 Jul 16];5(11):e138999. Available from: https://doi.org/10.1172/jci. insight.138999

24 Laridan E, Martinod K, De Meyer SF. Neutrophil extracellular traps in arterial and venous thrombosis. Semin Thromb Hemost. 2019 [cited 2020 Jul 19];45(1):86-93. Available from: https://pubmed.ncbi.nlm.nih. gov/30634198/ 